\title{
Development of Bio-Machine Based on the Plant Response to External Stimuli
}

\author{
K. Aditya, ${ }^{1,2}$ Ganesha Udupa, ${ }^{1}$ and Yongkwun Lee ${ }^{2}$ \\ ${ }^{1}$ Department of Mechanical Engineering, Amrita Vishwa Vidyapeetham, Amritapuri Campus, Kollam 690525, India \\ ${ }^{2}$ Centre for Bionics, Korea Institute of Science and Technology, Seoul 136-791, Republic of Korea
}

Correspondence should be addressed to Yongkwun Lee, yklee@kist.re.kr

Received 9 August 2011; Revised 31 October 2011; Accepted 11 November 2011

Academic Editor: Ivo Bukovsky

Copyright (C) $2011 \mathrm{~K}$. Aditya et al. This is an open access article distributed under the Creative Commons Attribution License, which permits unrestricted use, distribution, and reproduction in any medium, provided the original work is properly cited.

\begin{abstract}
In the area of biorobotics, intense research work is being done based on plant intelligence. Any living cell continuously receives information from the environment. In this paper, research is conducted on the plant named descoingsii $\mathrm{x}$ haworthioides (Pepe) obtaining the action potential signals and its responses to stimulations of different light modes. The plant electrical signal is the reaction of plant's stimulation owing to various environmental conditions. Action potentials are responsible for signaling between plant cells and communication from the plants can be achieved through modulation of various parameters of the electrical signal in the plant tissue. The modulated signals are used for providing information to the microcontroller's algorithm for working of the bio-machine. The changes of frequency of action potentials in plant are studied. Electromyography (EMG) electrodes and needletype conductive electrodes along with electronic modules are used to collect and transform the information from the plant. Inverse fast Fourier transform (IFFT) is used to convert signal in frequency domain into voltage signal for real-time analysis. The changes in frequency of the plant action potentials to different light modes are used for the control of the bio-machine. This work has paved the way for an extensive research towards plant intelligence.
\end{abstract}

\section{Introduction}

Much like humans, animals and plants have electrical signals which pass through them, but plants do not have nerves like humans and animals. Sanderson [1] was the first to discover the action potentials (APs) in the stimulation of a Dionaea leaf. Hence, electrical signals do not belong only to animal kingdom and humans [1]. Darwin [2] also found the response of some carnivorous venus fly trap plants [2]. Generally in humans and animals when the muscle is voluntarily contracted, action appears. In plants it is found that action potentials are the signals caused by the depolarization of plasma membrane [3, 4]. Green plants are able to show different electrical activity, which has been known long time ago [5]. Moreover, exhaustive studies in this field began only in the last decades of the former century along with the different contemporary experimental methods $[6,7]$. Electrical phenomena in plants have complicated character. Plant tissues are very complicated, highly structured consisting of both conductive and insulative elements. Because of that the resistance of different plants is not only ohmic but also frequency dependent.

In 1926, Bose used the isolated vascular bundles of a fern (Blechnum nudum) to show that excitation was transmitted as an electrical disturbance that appeared to be controlled by similar physiological events as in animal nerves [8]. In 1968, Backster by using polygraph or "lie detector" can measure electrical resistance, and water would alter the resistance of the leaf $[9,10]$. Various studies have been carried out on the plant signal and its intelligence. Recently researchers found that the transfer of volatile organic compounds (VOCs) signals among the plants. The signal is released by the emitter plant, and it is transported, absorbed, and perceived by the receiver plant [11]. The fastest methods of long-distance communication between the plant tissues and the organs are bio-electrochemical or electrophysiological signals. The effectiveness of such long-distance communication is clear, since plants can respond to external stimuli (e.g., changes in temperature or osmotic environment, illumination level, 
wounding, cutting, mechanical stimulation, or water availability) and changes can be detected in the plant soon after the injury $[7,12]$. The velocities of the propagation of electrical signals that have values from $0.5 \mathrm{~mm}$ to $4000 \mathrm{~mm}$ per second are sufficiently high to facilitate rapid longdistance communication, and these account for the rapid response phenomena observed in plants. The speed of propagation and the amplitude of action potential depend on the type of external stimulus [13]. It is also found that weak electrical signals of the chrysanthemum plant were tested by a touching test system of self-made double shields with platinum sensors [14]. Several studies have reported the effect of different stimuli that induce action potential gradients in plants, mainly light/dark $[15,16]$ temperature variations $[17,18]$, intense cold $[19,20]$, water availability $[21,22]$, mechanical wounding [23], and insects [22]. Also, it has been suggested that electrical signals could induce genetic programming $[24,25]$. After the electrical signal is produced, it is transmitted through the plant to a specific organ or tissue, which generates immediate physiological actions in response to the stimulation [26]. Floranium lamp was developed to measure the voltage signal of the plant [27]. The Daisy team is working on the "The PLANTS" project that will enable the plant to control its own environment [28]. Ivanhenriques developed a Jurema action plant with electrodes clamped to the plant branches [29].

1.1. Types of Signals in the Plant. Two types of signals in the plant have been described: fast signals (action potentials, APs) and slow signals (variation potentials, VPs). A new type of electrical potential signals, called system potentials, has been postulated recently. The novel "system potentials" were detected in five different plant species, among them agricultural crops like tobacco (Nicotian atabacum), maize (Zea mays), Barley (Hordeum vulgare), and field bean (Vicia faba) [20]. Action potentials are an electrical waveform that is transmitted along the cell membrane [30], characterized by a response. Plants respond to the environment change according to its amplitude, frequency, and intensity $[31,32]$. Thus, action potentials allow cells, tissues, and organs to transmit electrical signals over short and long distances in plants.

Variation potentials propagate in the plant, as temporal changes in the depolarization and repolarization of the cell membrane; this kind of signal varies with the intensity of stimulation and appears to be associated with changes in water tension or ion concentrations, creating a transient electrochemical unbalance in the xylem $[8,33]$.

1.2. Importance of Wounding Plant Leaf. If plant leaf is wounded, its action potential signal is stronger than that of unwounded leaf as shown in Figure 1 [20]. The strength of the inducing stimulus (wound signal) can influence the frequency when compared to that of systematic signal (unwounded signal).

In this research we are reporting a simple way for measuring the action potentials from the plant which is an approach different from those applied by earlier researchers.

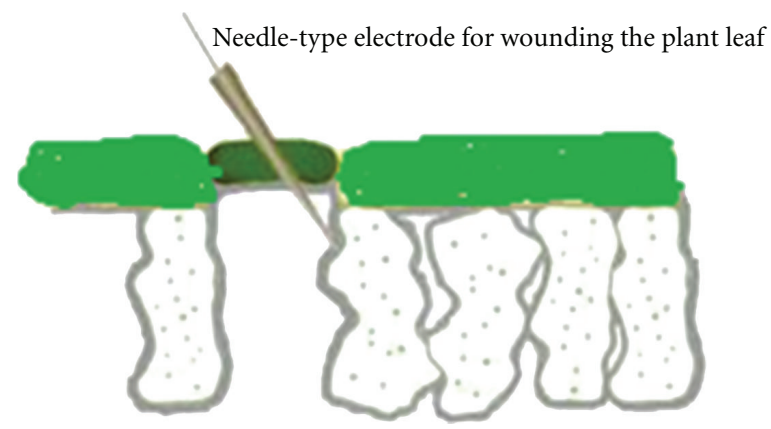

FIGURE 1: Insertion of electrodes through the stomata (small pores; the leaf is in dark green color) into the inner tissue. Plant electrical signal behavior can be changed by this process.

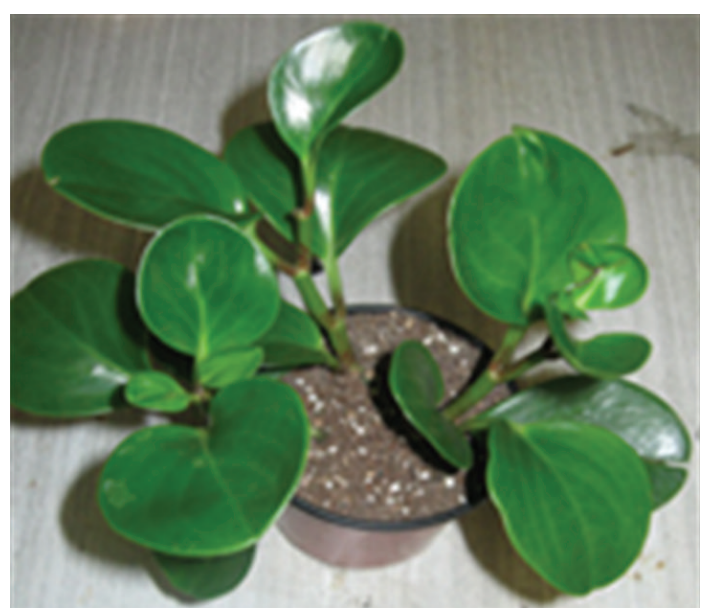

FIGURE 2: descoingsii $\mathrm{x}$ haworthioides plant for measuring the action potentials.

The speed of propagation duration and amplitude of action potentials depend on the location of the working electrodes and the reference electrode. The changes in frequency in the plant action potentials when it is exposed to different light modes are observed and used to control the bio-machine.

\section{Materials and Methods}

2.1. Plant and Laboratory Conditions. Different kinds of plants such as cactus (Cactaceae), soya beanplant (Glycine max), chrysanthemum (Dendranthema x grandiflorum), and Pepe (descoingsii $\mathrm{x}$ haworthioides) are purchased from the flower market and cultivated in the lab. It is observed that cactus is not responding and not showing any signal changes to the stimulus. The soybean and chrysanthemum plants are giving signals which are very weak to capture. The leaves of these plants are very thin, and it is difficult to injure them using the needle electrodes. The leaves of the descoingsii $\mathrm{x}$ haworthioides plant are very thick compared to the other plant leaves and sensitive to external stimulation. Hence descoingsii $\mathrm{x}$ haworthioides is selected for further research.

It is well known that plants follow diurnal cycle [15]. We assumed that plants are inactive during night time and rest 


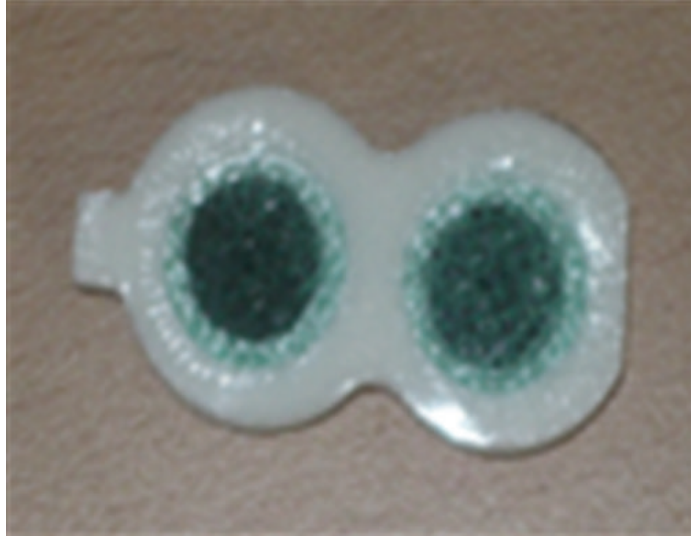

(a)

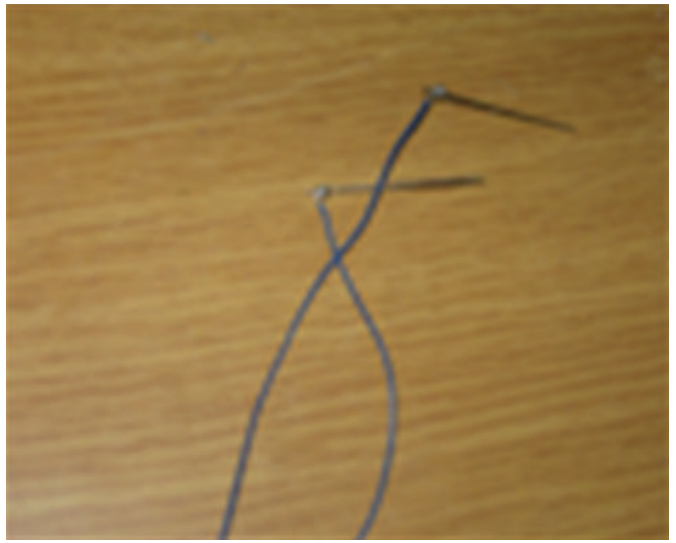

(b)

Figure 3: (a) Uni-Patch Tyco EMG electrodes (b) needle-type conductive electrodes.

like human beings, and hence diurnal cycle is not considered during the experiment. Experiments are conducted during morning, afternoon, and evening, and it was found that there is no much variation in the results since the experiments are conducted in an air-conditioned room with almost similar environment conditions for all the light modes.

In Figure 2 descoingsii $\mathrm{x}$ haworthioides plant is grown in a flower pot. The laboratory in which the experiments are carried out is a room with windows, and the temperature is kept at $26^{\circ} \mathrm{C}$ and the humidity at $75 \%$. The light in the room is turned off at 23:00 hrs, creating total darkness. In the morning once again the plant is exposed to normal room conditions. The plant is watered every alternate days.

2.2. Selection of Electrodes. Electromyography (EMG) electrodes are generally used to detect the electric potentials generated by muscle cells when these cells are electrically or neurologically activated. Two kinds of electrodes are used for detecting the signals coming from the plant.

First one is the Patch EMG electrode and the other is conductive needle-type electrodes. Uni Patch Tyco EMG electrodes which are sensitive and circular in shape with diameter of $20 \mathrm{~mm}$ and are used to collect the output signal from the plant leaves are shown in Figure 3(a). Another kind of electrodes used is needle-type conductive electrodes as shown in Figure 3(b), and it is made of copper. The length of the electrode is $350 \mathrm{~mm}$ and the edge of the electrode is very sharp. The surface area of the leaf is nearly $2600 \mathrm{~mm}^{2}$, and the area of electrode is nearly $314 \mathrm{~mm}^{2}$. The ratio of leaf surface area to electrode area is nearly $8: 1$. Figure 4 shows the connection of these electrodes to the plant. EMG electrode is kept away at a distance of $250 \mathrm{~mm}$ from the needle-type electrode to observe the action potentials.

\section{Experiments to Measure Electrical Signal from the Plant Leaf}

3.1. Experiment I. The objective of this experiment is to verify the behavior of action potentials in the plant. NI

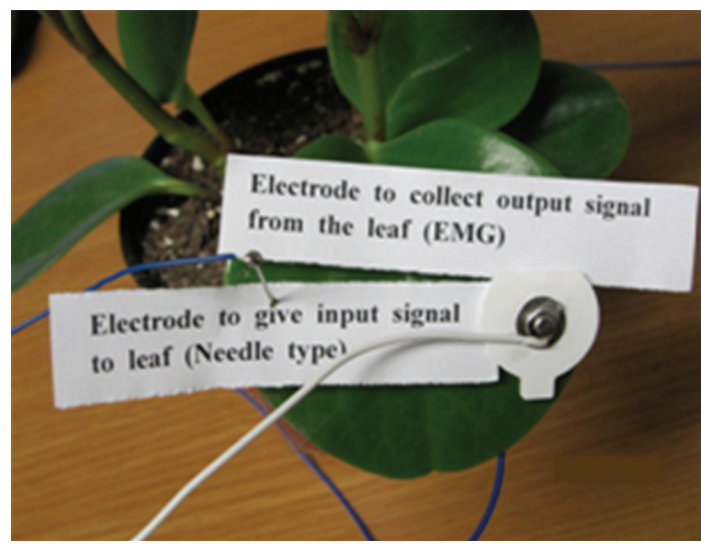

FIGURE 4: Insertion of EMG electrode and needle-type conductive electrode (by wounding the leaf).

LabVIEW is used for analyzing the signals coming from the plant.

3.1.1. Description of the Circuit. Totally four EMG electrodes and two needle-type conductive electrodes are used for the experiment. Three leaves of descoingsii $\mathrm{x}$ haworthioides plant are used in this experiment. Two leaves are used for the input (sinusoidal) signal from the function generator through two needle-type electrodes. The output signal is collected through two EMG electrodes for detecting the action potential signals from the plant and stored in data acquisition system. The other two EMG electrodes are used as reference electrodes connecting to each leaf. Figure 5 shows the hierarchy of the system for experiment I which shows the block diagram of the system components. The plant receives an input signal from the function generator, and the output signal from the plant is collected by using EMG electrodes and the data is stored in data acquisition system. The frequency of the function generator is chosen based on the action potential signal strength coming from the plant leaf. Figure 6 shows the experimental setup. 


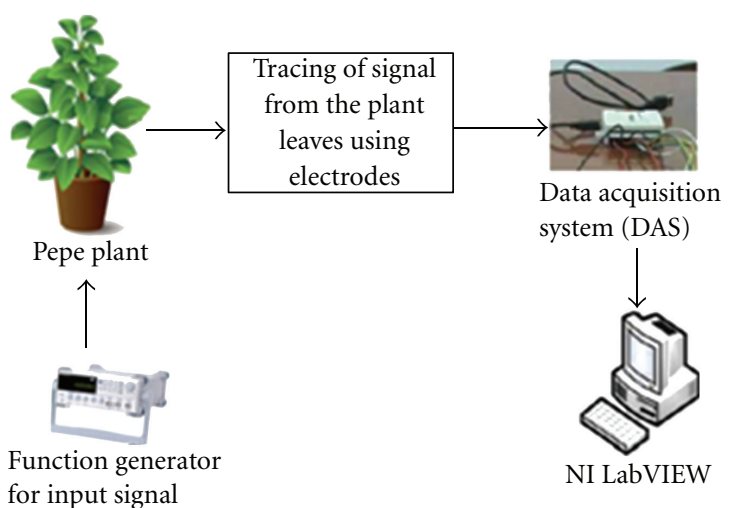

FIGURE 5: Hierarchy of system for experiment I.

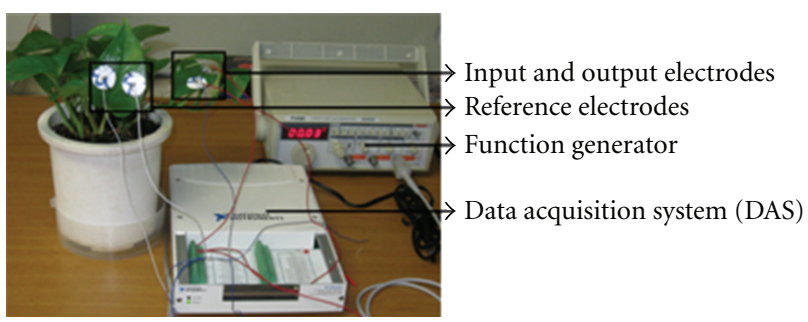

Figure 6: Apparatus for measuring the action potentials from the plant leaves.

3.1.2. Results and Discussion. The experiments are carried out with different frequencies ranging from $16 \mathrm{Khz}$ to $24 \mathrm{Khz}$. The output from the EMG electrode is connected to the NIDAS (data acquisition system). The analog output signal is viewed on the computer screen, and the same is converted to digital data and stored in the DAS. When the sinusoidal signal frequency is at $22 \mathrm{Khz}$ in the function generator, the output signal from the plant has the same nature as that of input signal but with little variation in amplitude as shown in Figure 7. In Figure 7, output signal from the two leaves of the plant is shown for a given input signal of the same frequency. This gives information about the output signal from the plant for a given sinusoidal input signal. Other types of signals such as square and triangle signals from the function generator are also given as input signal to the plant leaf by varying the frequencies, but the output obtained for these types of signals are very weak, and there is no much variation in the amplitude. Hence sinusoidal signal is chosen for this experiment.

3.2. Experiment II. The objective of this experiment is to verify the response of the plant when exposed to different light modes. Figure 8 shows the hierarchy of the system for experiment II.

In this experiment only one leaf is used to measure the signal coming from the plant. One needle-type conductive electrode is used for giving input signal. Two EMG electrodes are used, one for collecting output signal and the other as a reference electrode. In Figure 9, only the input electrode

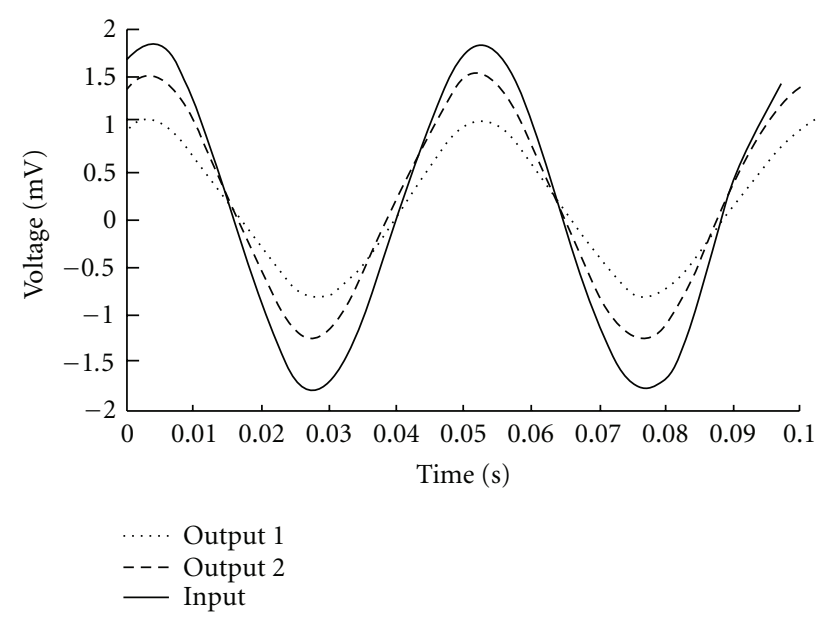

Figure 7: Output signals from the two leaves of the plant for a given sinusoidal input signal of same frequency.

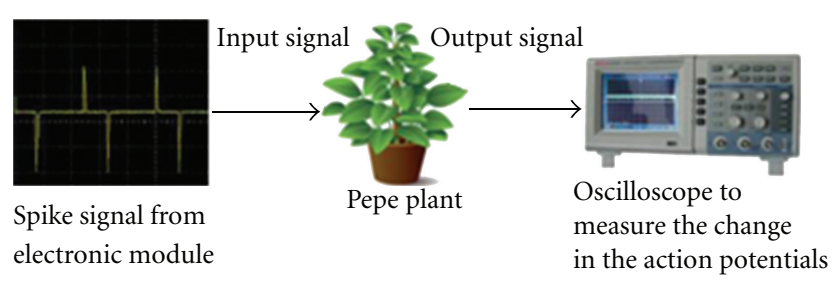

FIGURE 8: Hierarchy of system for experiment II.

and output electrode are shown, and the reference electrode connected to the ground is attached on the backside of the leaf. The glowing of LED is shown by a rectangular mark.

3.2.1. Description of the Circuit. Differential circuit is constructed by using NE555 timer to generate the spike signals. Figure 10 shows the circuit designed to get the spike signal as plant input signal so that change in the action potential signals from the plant leaf can be seen easily. These signals are very sharp and assist in detecting small changes in the amplitude of output signals. The voltage of input signal is $2.4 \mathrm{mV}$. The voltage of output signal collected from the plant leaf is $1.2 \mathrm{mV}$. The input frequency is $23.4 \mathrm{Khz}$, and the output frequency is $12.24 \mathrm{Khz}$. These readings are taken from the oscilloscope.

3.2.2. Procedure of the Experiment II. The input signal is given as spike signal from the electronic module shown in Figure 9, and the changes in action potential signals can be seen in oscilloscope.

The plant is exposed to three different light modes. The specification of the lamp used during experiments is GR2001 GRACE BIOLAMP, multifaceted reflector (MR16 HALOGEN LAMP). The brightness of the lamp can be adjusted. The maximum and minimum brightness of the lamp ranges between 560 lumens and 710 lumens, and medium brightness is 625 lumens. The mode of brightness of the table lamp is changed every 20 minutes. There is a 


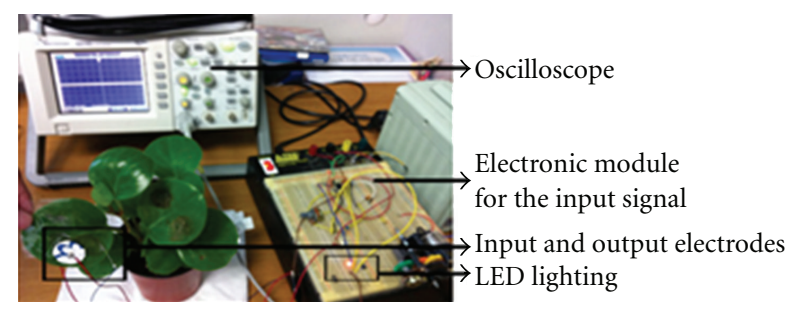

FIGURE 9: Apparatus for observing the change in action potential when plant is exposed to different light modes.

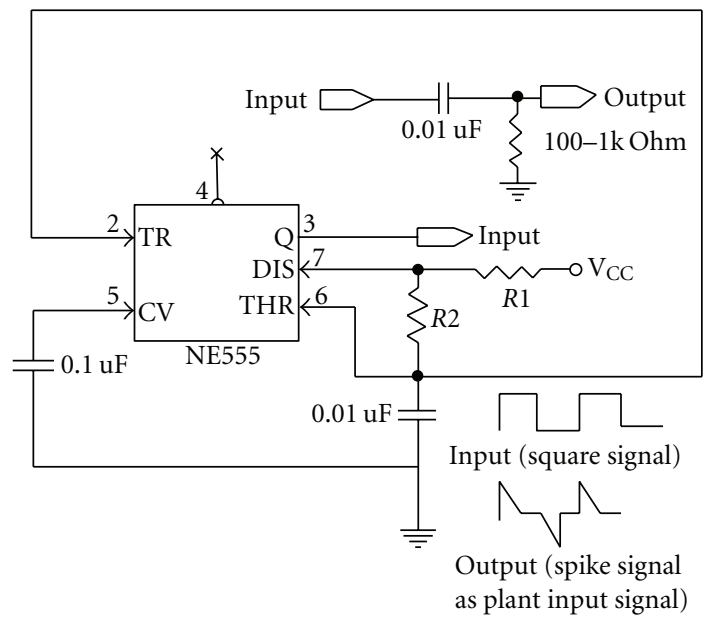

Figure 10: Circuit design for the plant input signal as spike signal.

change in frequency in the plant signal every 5 to $10 \mathrm{sec}$ during each measurement mode. Experiments are carried out for each light intensity mode for about 300 seconds (no light to maximum light brightness condition) after waiting for 30-45 minutes to stabilize the lamp intensity. The change in frequency of the action potential signal is observed from oscilloscope every 5 seconds using stop watch.

3.2.3. Results and Discussion. The observed maximum frequency values of the action potential signals in all the three modes of light are stored in the microcontroller. There is a little noise in the signal due to environmental vibrations. These effects are neglected since all the experiments are carried out in the similar environmental conditions. Figure 11 shows frequency responses of the action potential signals from the plant leaf taking into account all the three modes of light intensities.

There is an overlap in frequency values when there is a change in light intensity from no light to maximum brightness mode. These overlapped frequencies are removed during those particular time intervals by using the control algorithm developed for moving the bio-machine. The biomachine will move to the right, left or, straight based on the frequency values which are in close agreement with the values recorded in the microcontroller. Flow chart of the biomachine movement is described in Figure 12.

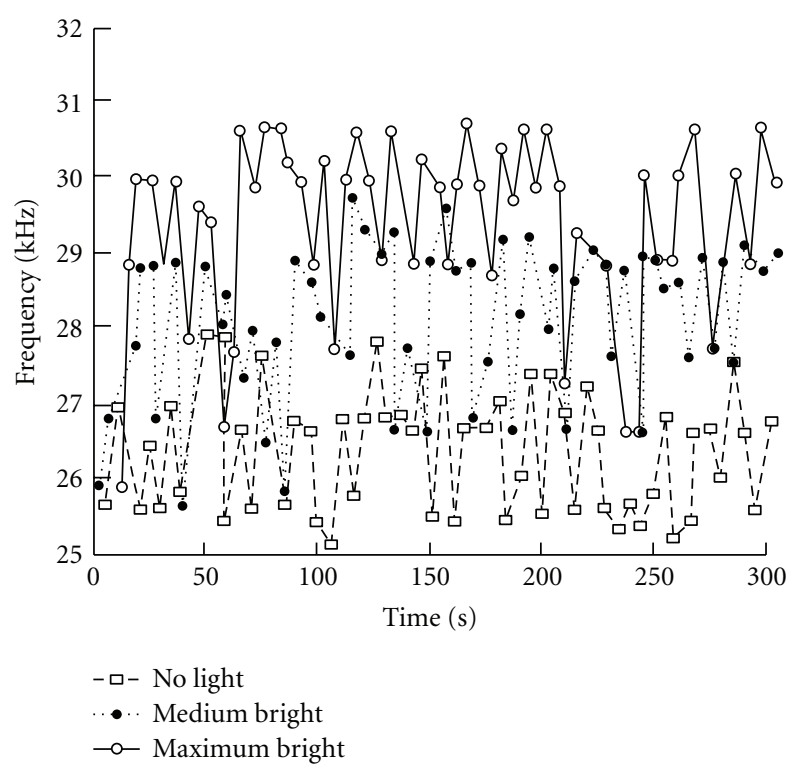

FIGURE 11: Frequency responses of the action potentials (APs) from the plant leaf at different time intervals when exposed to three modes of light intensities.

3.3. Converting Frequency to Voltage. By using the inverse fast Fourier transform (IFFT) in MATLAB (R2010b), the frequency values are converted to voltages as shown in Figure 13 for the three different light modes. The differences in the amplitudes can be clearly seen at around 150 seconds.

\section{Working and Control of Bio-Machine}

The change in frequencies when plant is exposed to different light conditions can be used as control signal for the bio-machine as shown in Figure 14. Inverse fast Fourier transform (IFFT) can be used to show dominant amplitudes in the three different light modes. These values are stored in microcontroller. Depending on the light modes, the biomachine will operate. Frequency counters can be used to see the response of plant to different light conditions, and the frequency can be converted into voltage signal by using the IC, LM2907. The direction change in the bio-machine can be seen by varying the light modes which results in the change in frequency of the action potential signals from the plant.

\section{Conclusion and Future Research}

In this research a simple method of detecting plant signals is investigated and the method is verified experimentally. The change in frequency levels is observed when the plant is exposed to different light conditions. Applying these results, bio-machine is constructed by designing a circuit and interfaced to a microcontroller. Software is written based on the developed algorithm to move the bio-machine in a desired way. There is a little inconsistency in the movement of the bio-machine due to the random nature of the signals. However, the present work has paved the way for extensive research on the plant intelligence in response to external 


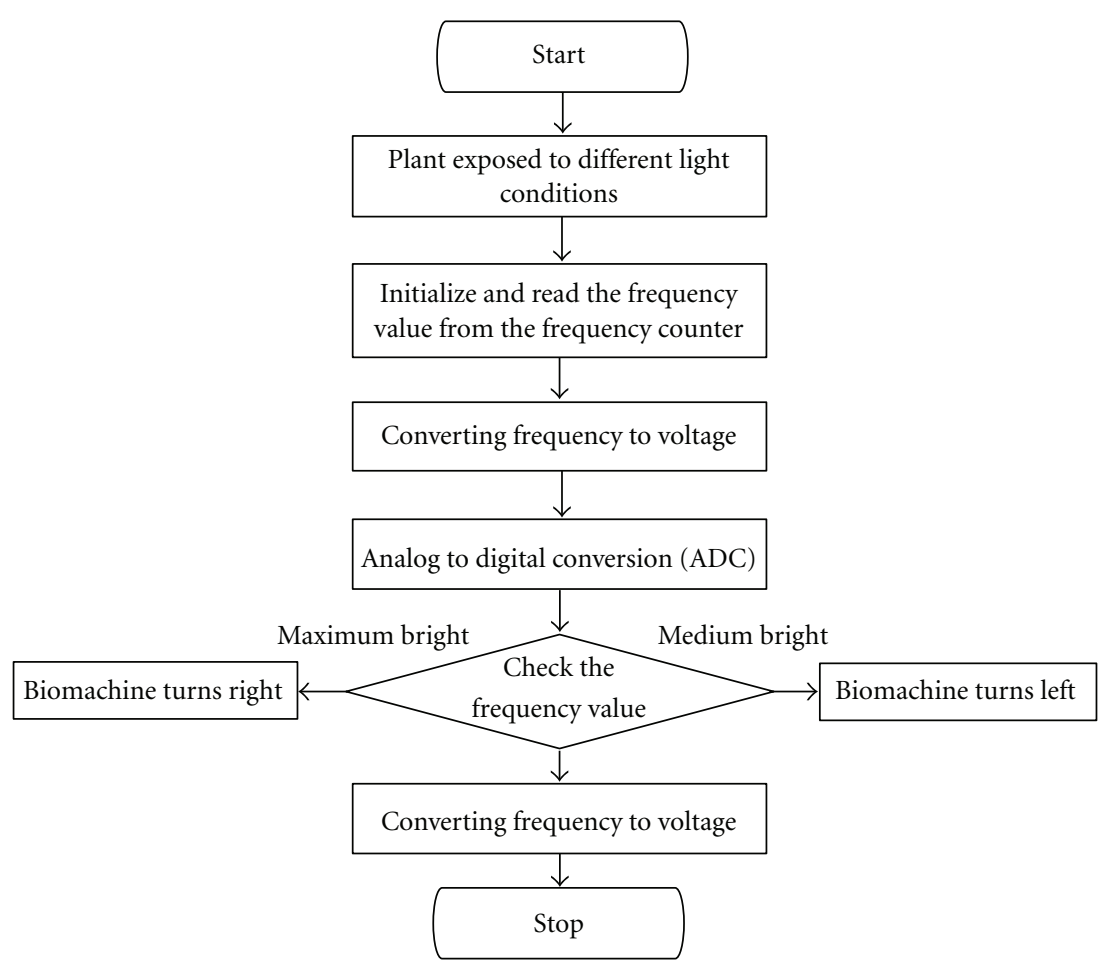

Figure 12: Flow chart for the movement of the bio-machine.

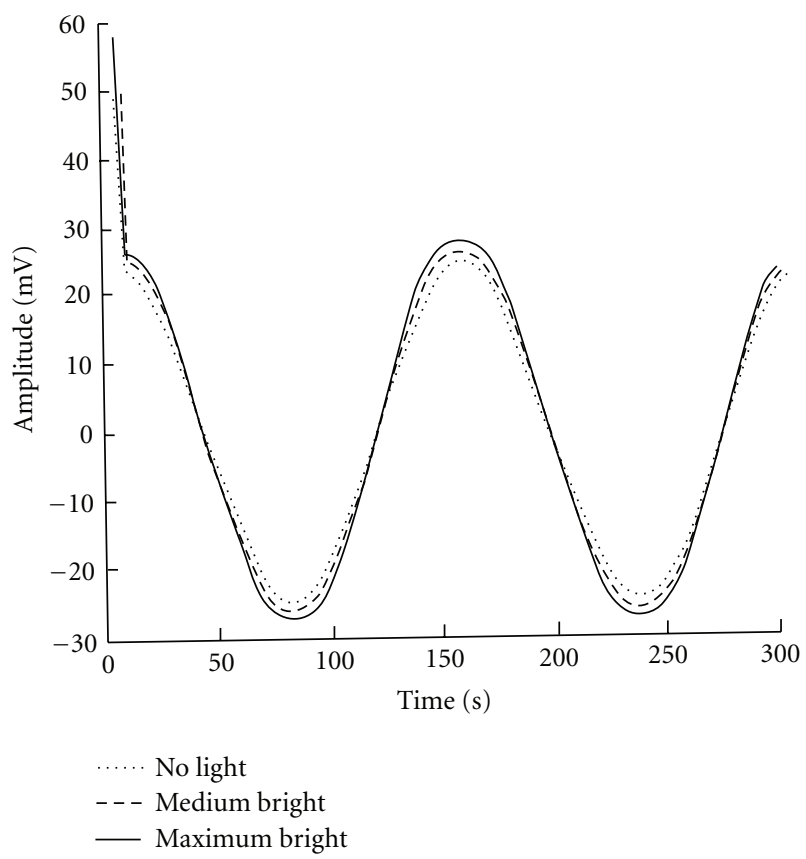

FIgURE 13: Real part of inverse fast Fourier transform (IFFT) for converting the frequency signal to amplitude $(\mathrm{mV})$.

stimuli as it holds out the true potential for innumerable and very interesting applications. Green plants interfaced with a computer through data acquisition systems can be used as biosensors for monitoring the environment and to detect the effects of pollutants on the plants. This method can

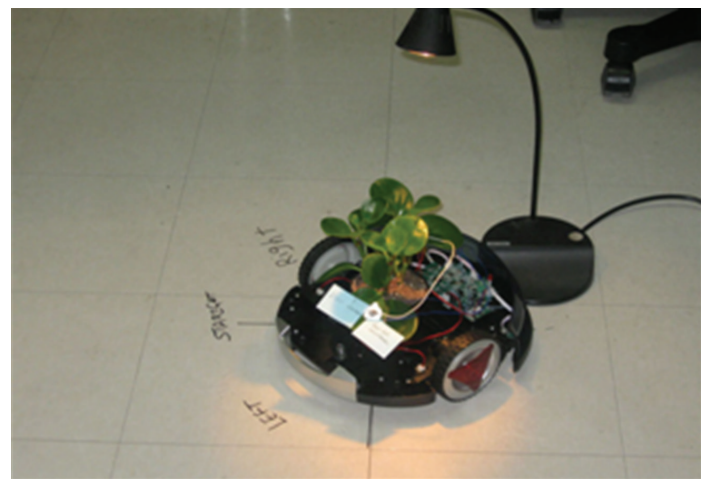

FIGURE 14: Prototype of bio-machine.

also be used for the study of plant electrophysiology. Future studies will be directed towards a better understanding of the plant action potential signals and its response to the other environmental conditions such as weather, presence of sunlight, temperature, use of pesticides and test for the better movement of the bio-machine in response to these environmental conditions.

\section{Acknowledgments}

The authors would like to thank the Korean Institute of Science and Technology (KIST) and Amrita Vishwa Vidyapeetham, Amrita School of Engineering, Amritapuri campus, for providing support to carry out the research and experiments. 


\section{References}

[1] J. Burdon Sanderson, "Note on the electrical phenomena which accompany irritation of the leaf of Dionaea muscipula," Royal Society of London Proceedings I, vol. 21, pp. 495-496, 1872.

[2] C. Darwin, Insectivorous Plants: John Murray, 1875.

[3] J. Fromm, "Control of phloem unloading by action potentials in Mimosa," Physiologia Plantarum, vol. 83, no. 3, pp. 529-533, 1991.

[4] J. Fromm and T. Bauer, "Action potentials in maize sieve tubes change phloem translocation," Journal of Experimental Botany, vol. 45, no. 273, pp. 463-469, 1994.

[5] M. Malone and B. Stankovi, "Surface potentials and hydraulic signals in wheat leaves following localized wounding by heat," Plant, Cell \& Environment, vol. 14, no. 4, pp. 431-436, 1991.

[6] D. Wildon, J. Thain, P. Minchin et al., "Electrical signalling and systematic proteinase inhibitor induction in the wounded plant," Nature, vol. 360, no. 6399, pp. 62-65, 1992.

[7] O. S. Ksenzhek and A. G. Volkov, Plant Energetics, Academic Press, 1998.

[8] S. J. C. Bose, The Nervous Mechanism of Plants, Longmans Green, London, UK, 1926.

[9] C. Backster, "Evidence of a primary perception in plant life," International Journal of Parapsychology, vol. 10, no. 4, pp. 329$348,1968$.

[10] S. Lautner, T. E. E. Grams, R. Matyssek, and J. Fromm, "Characteristics of electrical signals in poplar and responses in photosynthesis," Plant Physiology, vol. 138, no. 4, pp. 2200 2209, 2005.

[11] I. T. Baldwin, R. Halitschke, A. Paschold, C. C. Von Dahl, and C. A. Preston, "Volatile signaling in plant-plant interactions: "talking trees" in the genomics era," Science, vol. 311, no. 5762, pp. 812-815, 2006.

[12] A. G. Volkov and R. A. Haack, "Insect-induced bioelectrochemical signals in potato plants," Bioelectrochemistry and Bioenergetics, vol. 37, no. 1, pp. 55-60, 1995.

[13] A. G. Volkov, T. C. Dunkley, A. J. Labady, and C. L. Brown, "Phototropism and electrified interfaces in green plants," Electrochimica Acta, vol. 50, no. 21, pp. 4241-4247, 2005.

[14] L. Wang and Q. Li, "ARIMA signals processing of information fusion on the chrysanthemum," Lecture Notes in Computer Science, vol. 6319, no. 1, pp. 239-247, 2010.

[15] P. Datta and P. Palit, "Relationship between environmental factors and diurnal variation of bioelectric potentials of an intact jute plant," Current Science, vol. 87, no. 5, pp. 680-683, 2004.

[16] L. A. Gurovich and P. Hermosilla, "Electric signalling in fruit trees in response to water applications and light-darkness conditions," Journal of Plant Physiology, vol. 166, no. 3, pp. 290-300, 2009.

[17] J. D. Rhodes, J. F. Thain, and D. C. Wildon, "The pathway for systemic electrical signal conduction in the wounded tomato plant," Planta, vol. 200, no. 1, pp. 50-57, 1996.

[18] S. Pyatygin, V. Opritov, and V. Vodeneev, "Signaling role of action potential in higher plants," Russian Journal of Plant Physiology, vol. 55, no. 2, pp. 285-291, 2008.

[19] E. D. Brenner, R. Stahlberg, S. Mancuso, J. Vivanco, F. Baluska, and E. Van Volkenburgh, "Plant neurobiology: an integrated view of plant signaling," Trends in Plant Science, vol. 11, no. 8, pp. 413-419, 2006.
[20] M. R. Zimmermann, H. Maischak, A. Mithöfer, W. Boland, and H. H. Felle, "System potentials, a novel electrical longdistance apoplastic signal in plants, induced by wounding," Plant Physiology, vol. 149, no. 3, pp. 1593-1600, 2009.

[21] J. Fromm and H. Fei, "Electrical signaling and gas exchange in maize plants of drying soil," Plant Science, vol. 132, no. 2, pp. 203-213, 1998.

[22] T. E. E. Grams, C. Koziolek, S. Lautner, R. Matyssek, and J. Fromm, "Distinct roles of electric and hydraulic signals on the reaction of leaf gas exchange upon re-irrigation in Zea mays L," Plant, Cell and Environment, vol. 30, no. 1, pp. 79-84, 2007.

[23] A. Schaller and C. Oecking, "Modulation of plasma membrane $\mathrm{H}^{+}$-ATPase activity differentially activates wound and pathogen defense responses in tomato plants," The Plant Cell, vol. 11, no. 2, pp. 263-272, 1999.

[24] F. Baluška, S. Mancuso, D. Volkmann, and P. Barlow, "Root apices as plant command centres: the unique "brain-like" status of the root apex transition zone," Biologia, vol. 59, supplement P, pp. 7-19, 2004.

[25] F. Baluska, D. Volkmann, and D. Menzel, "Plant synapses: actin-based domains for cell-to-cell communication," Trends in Plant Science, vol. 10, no. 3, pp. 106-111, 2005.

[26] B. Stanković and E. Davies, "The wound response in tomato involves rapid growth and electrical responses, systemically up-regulated transcription of proteinase inhibitor and calmodulin and down-regulated translation," Plant and Cell Physiology, vol. 39, no. 3, pp. 268-274, 1998.

[27] FLORANIUM, "LightArtVision,” 2011, http://www.floranium .com/index.php.

[28] “The PLANTS PROJECT,” DaisyTeam 2011, http://daisy.cti .gr/plants/.

[29] Ivanhenriques, "JuremaActionPlant," Exhibition Taming Technology, Florence (IT) 2011, http://ivanhenriques.wordpress.com/.

[30] A. G. Volkov, T. C. Dunkley, S. A. Morgan, D. Ruff, Y. L. Boyce, and A. J. Labady, "Bioelectrochemical signaling in green plants induced by photosensory systems," Bioelectrochemistry, vol. 63, no. 1-2, pp. 91-94, 2004.

[31] J. Fromm and S. Lautner, "Electrical signals and their physiological significance in plants," Plant, Cell and Environment, vol. 30, no. 3, pp. 249-257, 2007.

[32] A. Volkov and C. Brown, "Electrochemistry of plant life," Plant Electrophysiology, pp. 437-459, 2006.

[33] J. Mwesigwa, D. J. Collins, and A. G. Volkov, "Electrochemical signaling in green plants: effects of 2, 4-dinitrophenol on variation and action potentials in soybean," Bioelectrochemistry and Bioenergetics, vol. 51, no. 2, pp. 201-205, 2000. 

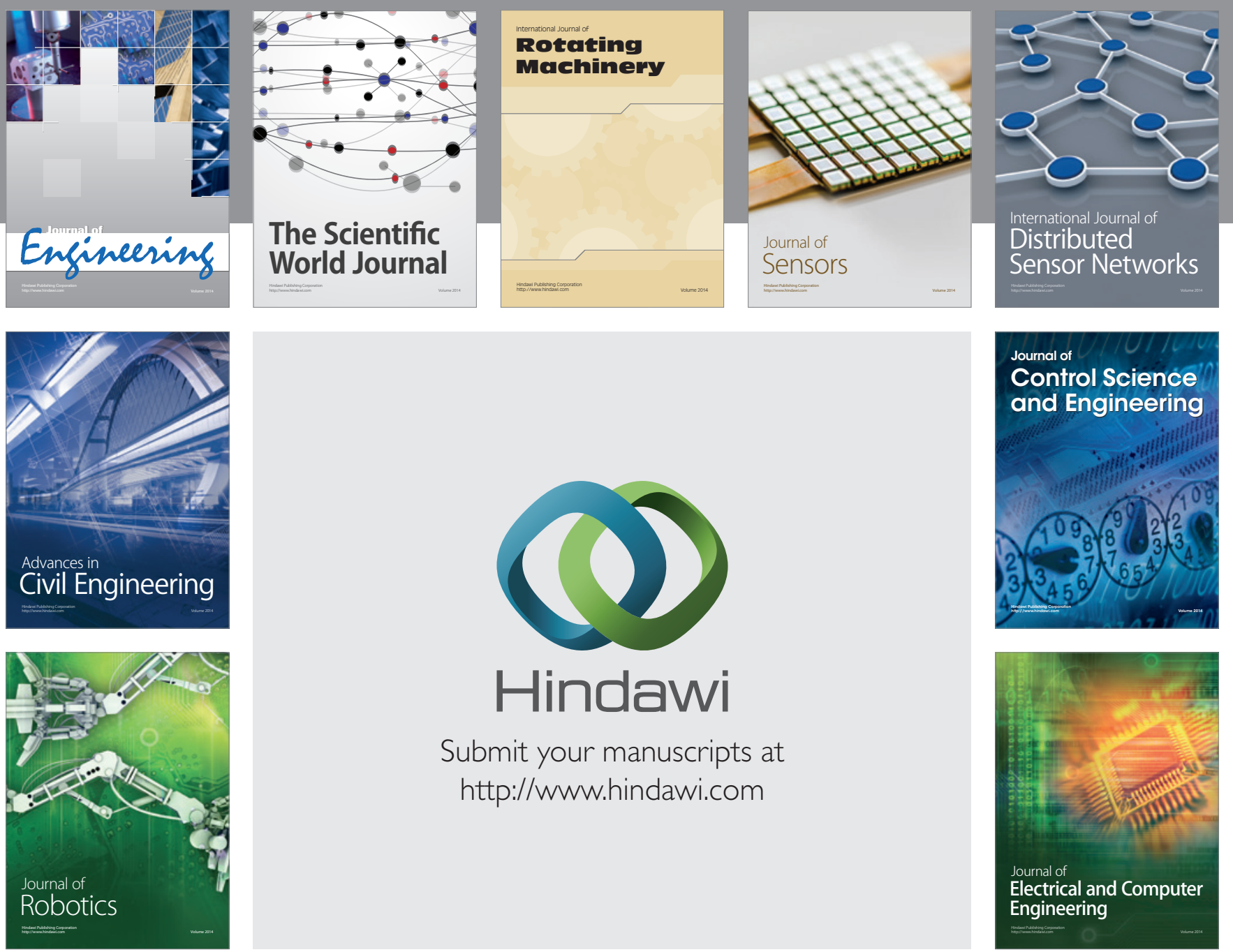

Submit your manuscripts at

http://www.hindawi.com
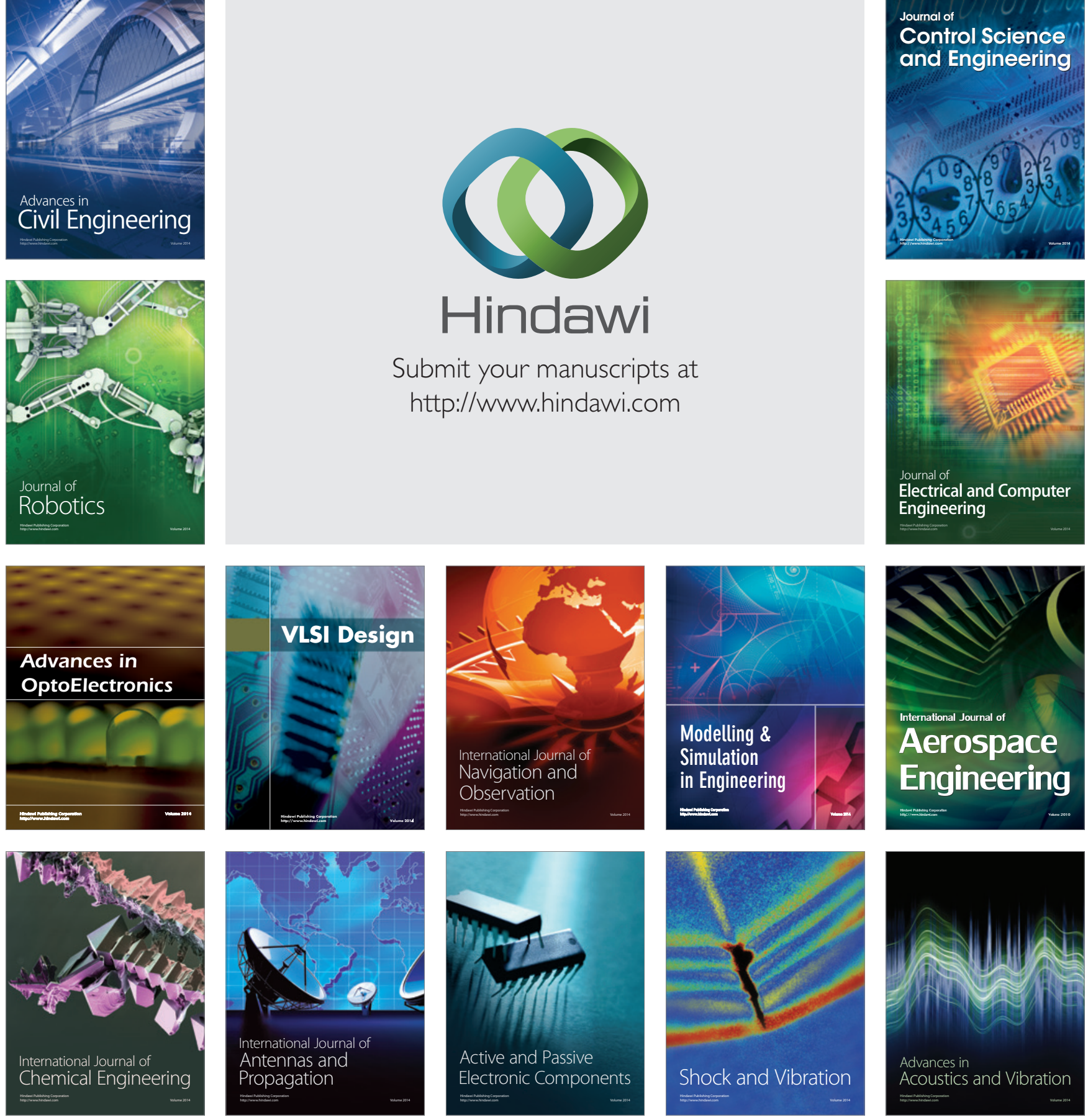